\title{
Simulating Bistable Perception with Interrupted Ambiguous Stimulus using Self-Oscillator Dynamics with Percept Choice Bifurcation
}

\section{Norbert Fürstenau}

German Aerospace Center, Inst. of Flight Guidance

Phone: -49 531295 2549, Fax: +49 5312952550

norbert.fuerstenau@dlr.de

A behavioral stochastic self-oscillator model with perception-attention coupling and re-entrant cognitive processing is used for simulating interrupted ambiguous stimulus induced percept reversals. The results provide further support for a dynamical systems foundation of cognitive and psychological problems as discussed in detail within the context of Gestalt psychology [1], and for coordination dynamics of the brain [2]. Periodic stimulus-off switching ( $\mathrm{t}_{\mathrm{off}}<1 \mathrm{~s}, \mathrm{t}_{\mathrm{on}}=300 \mathrm{~ms}$ ) was introduced by Orbach et al. [3] as experimental paradigm to get more insight into the underlying perceptual dynamics. Their Necker cube experiments showed a maximum of the percept reversal rate $\mathrm{R}$ at $\mathrm{R}_{\max } \approx 36 \mathrm{~min}^{-1}$ and $\mathrm{t}_{\mathrm{off}} \approx 200 \mathrm{~ms}$ which was confirmed by recent experiments [4]. Noest et al. [5] demonstrated with a low level neural activation model [6] that a bifurcation of the percept choice dynamics during the ambiguous-stimulus on-off switching dominates the statistics of the reversal time series. Our simulations based on a macroscopic (behavioral) nonlinear dynamics model [7][8] (similar to [9]) support this finding. They show that the measured R vs. $t_{\text {off }}$-time characteristics can be fitted with only a few model parameters: Thalamo-cortical reentrant delay $\mathrm{T}=40 \mathrm{~ms}$, attention fatigue (= adaptive feedback gain) time constant $=1-2 \mathrm{~s}$, feedback-gain noise power $\mathrm{J}_{\omega}$. Synchronisation of attention fatigue induced self-oscillations (yielding inter-stimulus transition time $\mathrm{T}_{\mathrm{Tr}} \approx 4-5 \mathrm{~T}$ ) in combination with stimulus-onset induced percept choice bifurcation appears to determine the percept-reversal rates and the $t_{\text {off }}$-value at $R_{\max }$. A linear approximation of the dynamical equations allows for an analytical estimate of the reversal frequency and the cognitive damping time constant ( $\tau_{\mathrm{v}} \approx 1 \mathrm{~s}$ ). By use of the Fluctuation-Dissipation theorem via noise power $\mathrm{J}_{\omega}$ it defines an index of cognitive inertia (as suggested in [10]) as crucial parameter of the simulated dynamics.

[1]Wagemans,J., Feldman, J., Gepshtein, S., Kimchi, R., Pomerantz, J. R., van der Helm, P.A., van Leeuwen, C. (2012) A century of Gestalt Psychology in visual perception: II. Conceptual and theoretical foundations, Psychological Bulletin 138 (6), 1218-1252

[2] Kelso J.A.S. (2012): Multistability and Metastability: Understanding dynamic coordination in the brain. Phil Trans. R. Soc. B vol. 367 pp. 906-918

[3] Orbach. J., Zucker, E., Olson, R. (1966). Reversibility of the Necker Cube: VII. Reversal rate as a function of figure-on and figure-off durations. Percept. Motor Skills (22), 615-618

[4] Kornmeier, J., Ehm, W. Bigalke, H., Bach, M. (2007): Discontinuous presentation of ambiguous figures:

How interstimulus-interval durations affect reversal dynamics and ERP's. Psychophysiology, 44, 552-560

[5] Noest, A.J., van Ee, R., Nijs, M.M., van Wezel, R.J.A. (2007). Percept-choice sequences driven by interrupted ambiguous stimuli: A low-level neural model. J of Vision 7, 1-14

[6] Amari, S. (1977): Dynamics of pattern formation in lateral-inhibition type neural fields. Biological Cybernetics vol. 27, 77-87

[7] Fürstenau, Norbert (2010). A nonlinear dynamics model for simulating long range correlations of cognitive multistability. Biol. Cybern., vol. 103. (3) 175-198

[8] Fürstenau, Norbert (2014). Simulating Bistable Perception with Interrupted Ambiguous Stimulus using SelfOscillator Dynamics with Percept Choice Bifurcation. Cognitive Processing, 15 (4), 467-490 DOI:

10.1007/s10339-014-0630-4.

[9] Ditzinger, T., Haken, H. (1989). Oscillations in the Perception of Ambiguous Patterns. Biol. Cybern. ( 61) 279-287

[10] Gao, J.B., Merk, I., Tung W. W., Billok V., White, K.D., Harris J G, Roychowdhury V P. (2006). Inertia and memory in visual perception. Cogn. Processing vol. 7 105-112 